\title{
Interactive Virtual Relighting and Remodeling of Real Scenes
}

Céline Loscos $\dagger$, Marie-Claude Frasson $\dagger \neq$, George Drettakis $\dagger$,

Bruce Walter $\dagger$, Xavier Granier†, Pierre Poulin $\ddagger$

$\dagger i$ MAGIS-GRAVIR/IMAG-INRIA

B.P. 53, F-38041 Grenoble, Cedex 9, France

†Département d'informatique et de recherche opérationnelle, Université de Montréal

No 0230

Avril 1999

THÈME 3

\section{apport \\ technique}





\title{
Interactive Virtual Relighting and Remodeling of Real Scenes
}

\author{
Céline Loscos $\dagger$, Marie-Claude Frasson $\dagger \ddagger$, George Drettakis $\dagger$, \\ Bruce Walter $\dagger$, Xavier Granier $\dagger$, Pierre Poulin $\ddagger$ \\ $\dagger i$ MAGIS-GRAVIR/IMAG-INRIA \\ B.P. 53, F-38041 Grenoble, Cedex 9, France \\ †Département d'informatique et de recherche opérationnelle, Université de \\ Montréal
}

Thème 3 - Interaction homme-machine, images, données, connaissances Projet iMAGIS

Rapport technique n0230 - Avril 1999 - 16 pages

\begin{abstract}
Lighting design is tedious due to the required physical manipulation of real light sources and objects. As an alternative, we present an interactive system to virtually modify the lighting and geometry of scenes with both real and synthetic objects, including mixed real/virtual lighting and shadows.

In our method real scene geometry is first approximatively reconstructed from photographs. Additional images are taken with a real light in different positions from the same viewpoint to estimate reflectance. A filtering process is used to compensate for modeling errors, and per image reflectances are averaged to generate an approximate reflectance image for the given viewpoint, removing shadows in the process. This estimate is used to initialise a global illumination hierarchical radiosity system, representing real-world secondary illumination; the system is optimized for interactive updates. Direct illumination from lights is calculated separately using ray-casting and a table for efficient reuse where appropriate.

Our system allows interactive modification of light emission and object positions, all with mixed real/virtual illumination effects. Real objects can also be virtually removed using texture-filling algorithms for reflectance estimation.
\end{abstract}

Key-words: Image synthesis, computer augmented reality, virtual reality, reflectance estimation, common illumination, virtual relighting, virtual removal of real objects, hierarchical radiosity, global illumination, interactivity. 


\section{Rééclairage/Remodélisation Virtuels et Interactifs des Scènes Réelles}

Résumé : Le design de l'éclairage d'interieur est un travail laborieux, notamment à cause de la manipulation "physique" des lampes et des objets. En solution alternative, nous proposons un système interactif pour modifier virtuellement l'éclairage et la géometrie des scènes, comprenant à la fois des objets réels et virtuels, en tenant compte des éclairages mixtes (réels / virtuels) et des ombres.

Dans notre méthode, la géométrie de la scène réelle est d'abord approximativement reconstruite à partir de photos. Puis de nouvelles photos sont prises depuis le même point de vue, mais sous des éclairages différents, et servent à estimer la reflectance des objets réels. Un processus de filtrage est appliqué pour compenser les erreurs de modélisation. La moyenne des reflectances calculées pour chaque image, permet d'obtenir une image de reflectance pour le point de vue donné, enlevant ainsi les ombres. Cette estimation est utilisée pour initialiser un systéme de radiosité hiérarchique de simulation d'illumination globale, afin de représenter l'illumination indirecte réelle. Le système est optimisé pour favoriser des mises à jour interactives. L'éclairage direct dû aux lampes est calculé séparément par du lancer de rayon et une table est maintenue pour une réutilisation aux endroits apropriés.

Notre système permet une modification interactive de l'intensité des lampes et de la position des objets, en tenant compte des effets lumineux mixtes entre objets réels et virtuels. Les objets réels peuvent aussi être enlevés virtuellement en utilisant des algorithmes de remplissage de textures pour l'estimation de la reflectance.

Mots-clé : Synthèse d'images, réalité augmentée assistée par ordinateur, réalité virtuelle, estimation de la reflectance, éclairage commun, rééclairage virtuel, enlèvement d'objets réels, radiosité hiérarchique, éclairage global, interactivité. 


\section{Introduction}

Designing the illumination of real environments has always been a difficult task. Lighting design for home interiors for example, is a complex undertaking, requiring much time and effort with the manipulation of physical light sources, shades, reflectors, etc. to create the right ambiance. In addition other physical objects may need to be moved or otherwise changed. The problem is even more complex on movie sets or exterior lighting design. The fundamental trial-and-error nature of the relighting process makes it painful and often frustrating; more importantly, the requirements of constructing and moving real objects and light sources make testing many different potential designs often impossible.

Ideally, we would like to perform such processes entirely synthetically. The lighting designer would simply photograph the environment to be relit and/or remodeled, and then create the different conditions by computer simulation so they can be evaluated appropriately.

Evidently, such a goal is very hard to accomplish. In this paper we provide first solutions to a subset of this goal, inspired by techniques developed for computer augmented reality, and common illumination between the real and the synthetic scenes $[2,10]$.

Our method starts with a preprocess, in which real geometry is reconstructed from a series of photos [20], taken from several different viewpoints. A second set of images (which we call radiance images) are taken from a fixed viewpoint with a real light source in different positions. The geometry and the images are used to extract an approximate reflectance at each pixel for the given point of view. Intuitively, we attempt to have an unoccluded view of the light source at each pixel in at least one radiance image, which gives a good reflectance estimate; we compensate for geometric and photometric imprecision using a filtering step on individual radiance images before performing a weighted average. The result of this new approach is an acceptable estimate of reflectance, called a reflectance image; in the process, shadows are removed in a satisfactory manner.

Our main goal is to provide interactive manipulation of mixed real and virtual environments with common illumination. To achieve this we have separated the calculation of direct and indirect illumination. The reflectance image is used to initialise a hierarchical radiosity system with clustering [23], optimized for dynamic updates [6]. This structure is used for rapid updates of indirect light, while direct light is computed on a pixel-by-pixel basis. For direct light many components can be pre-computed and stored in a table for rapid modification, and in other cases the changes are limited to small regions of screen space, permitting interactive updates. Working on a pixel-by-pixel basis results in high quality direct shadows and also facilitates the removal of real objects, since we simply manipulate the reflectance image using texture generation methods.

It is important to note outright that we do not attempt to extract accurate reflectance values. The goal of our method is to achieve convincing relighting at interactive rates. To this end we can ignore inaccuracies and small artifacts, if the overall effect is believable.

\section{Previous work}

A large body of literature exists in computer vision on reconstructing 3D scenes from photos[8]. However the quality of the extracted 3D models has only recently become satisfactory for computer graphics applications with the presentation of interactive systems such as Photomodeler[19], REALISE[9, 15], Façade[4], and others[20]. While they all include some form of texture extraction and mapping, none treat the extraction of surface properties and re-illumination. Sato et al.[21] present a system to extract 3D geometry, texture, and surface reflectance, but it is limited to a very controlled environment.

RT n ${ }^{\circ} 0230$ 
With the development of an ever increasing number of computer augmented reality applications, it becomes important to handle the common illumination between real and synthetic scenes. While some previous papers $[10,5]$ present preliminary solutions, they all require significant user intervention and are limited in different ways in the lighting or geometric conditions they can treat. Recent developments to Façade[2] include surfaces property extraction, but rendering times of the Radiance [24] system used for image-generation are far from interactive.

Nakamae et al. [18] developed a solution for merging virtual objects into background photographs, and estimated the sun location to simulate common illumination effects in outdoor environments. More recently Yu and Malik [27] proposed a solution to virtually modify the illumination with different virtual positions of the sun in outdoor scenes.

Loscos and Drettakis [16, 17] have developed an approach to remove shadows, thus enabling synthetic relighting. This technique attempts to remove shadows by computing the best possible approximation using a single image. Despite successful results for certain cases, certain visual artifacts remain in the shadow regions.

In our method, as mentioned in the introduction, we separate direct lighting, which can be easily computed for each pixel, from indirect, or global lighting. Since we will be interactively modifying the scene, we need to be able to update the global illumination rapidly. To do this, we have used some of the ideas developed by Shaw [22] and Drettakis and Sillion [6].

Removal of real objects from a reconstructed scene requires some form of hole-filling in the real images/textures containing the real objects being removed. Heeger and Bergen [13] have developed a method to synthesize texture images given a texture sample. They use a series of linear filters to analyse the sample and create a texture that matches the sample appearance. Their method is successful on "stochastic" textures (e.g. stucco) but fails on "deterministic" textures (e.g. bricks). El-Maraghi [7] has provided a public domain implementation of their algorithm.

Igehy and Pereira [14] integrate a composition step into Heeger and Bergen algorithm in order to "erase" flaws (e.g. stains or undesired features) from images. They manually create a mask which indicates which part of the image is to be covered by the synthesized texture and which part keeps its original texture.

\section{Overview of the Method}

Our goal is to allow interactive synthetic relighting and remodeling of real environments including both removing real lights or objects, and adding virtual ones. To accomplish this, we need to build approximate geometric and reflectance models of the environment and quickly estimate the illumination in modified configurations. We also want our method to be tolerant of measurement and modeling errors in order to work on a broad class of environments. Our process consists of several preprocessing steps followed by an interactive relighting session.

We begin by taking two sets of photographs of the target environment. The first is taken from multiple viewpoints under normal lighting conditions and is used to build an approximate geometric model of the model using our photomodeling system[20]. The second set is taken from the fixed viewpoint that will be used during the interactive editing session. These photos use controlled lighting which consists of a single known light source that is moved between photos. We typically use between 5 and 7 such photos such as those in Figure 1. This second set, which we will refer to as the radiance images, is used to estimate the reflectance on all the visible surfaces.

The geometric and reflectance models are used to initialize an optimized hierarchical radiosity system that is used to dynamically simulate the indirect lighting in the environment. To recreate

INRIA 


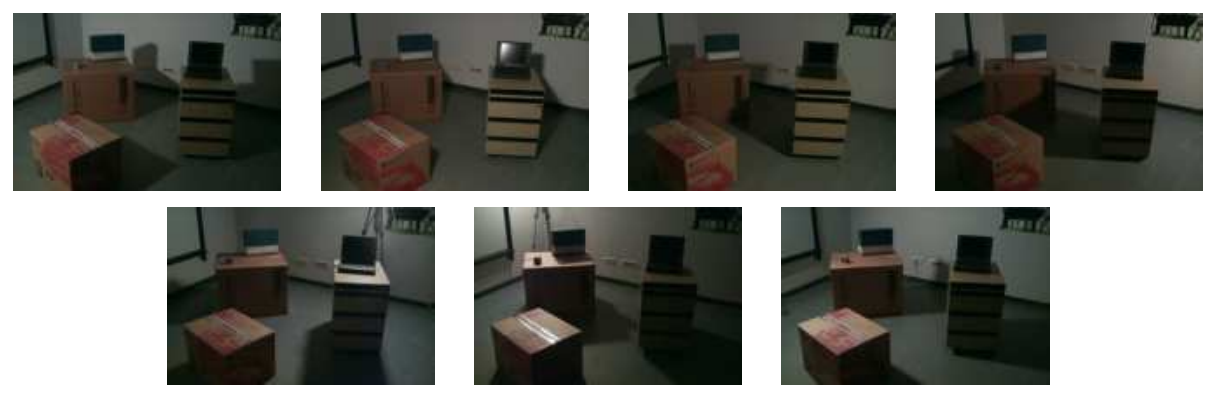

Figure 1: The 7 radiance images used for the example presented in this paper.

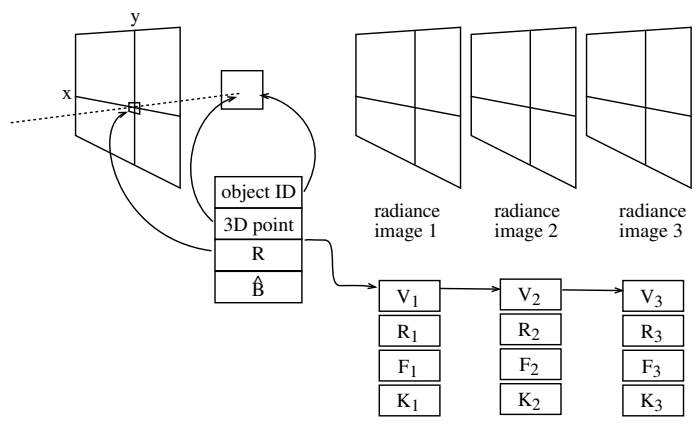

Figure 2: A per pixel data structure is stored for the interactive view as well as for each radiance image. The visibility to each source $V_{i}$, the form factor to each light $F_{i}$, and the estimated reflectance at this pixel $R$. The interactive view also stores the surface id and 3D point corresponding to each pixel.

sharp shadows, the direct lighting is estimated on a per pixel basis from the fixed viewpoint using ray casting. For each pixel we store its corresponding 3D point and surface, its estimated local reflectance, and its visibility and form factors to each light. This structure is illustrated in Figure 2.

Each single radiance image is used to estimate all the pixel reflectances, but may be unreliable in some regions such as shadows. We generate a more robust reflectance estimator by assigning confidence in each estimate and combining them from the multiple images accordingly.

After approximating reflectance in visible regions, we also estimate the reflectance in regions that might become visible due to object removal. This is accomplished by adapting a texture-filling algorithm. Once the radiosity and per pixel data structures have been initialized, we are ready to interactively remodel and relight our scene.

\section{Preprocessing}

The main goal of the preprocessing steps is to initialize the data structures that will be used during the interactive session. First surface reflectance at each pixel is estimated, and a pixel-based data structure for precomputed direct lighting quantities is initialized. Finally the hierarchical radiosity system is setup for rapid indirect lighting updates.

The process begins by building a geometric model of the environment using our photomodeling system [20]. The user specifies a set of corresponding points in the set of photographs taken from multiple viewpoints. The system uses these to solve for the camera parameters and $3 \mathrm{D}$ positions of $\mathrm{RT} \mathrm{n}^{\circ} 0230$ 
the points. The user connects these points together into polygons to form a geometric model of the scene and can specify additional constraints to improve the model. All further processing uses the radiance images, and the position of the light source is measured by hand.

\subsection{Pixel Data Structure}

The radiance images are all taken from a single viewpoint which is the same viewpoint that we will use in our interactive remodeling session. The physical light source we used is a simple garden light with a base, covered by white semi-transparent paper to achieve a more diffuse effect. Using a fixed viewpoint simplifies the capture of the real scene (fewer images); in addition working in image space allows more efficient data structures to be used for display, and generally simplifies the algorithms developed.

Much of the computation is done on a per pixel basis based on this viewpoint using an augmented pixel data structure. At each pixel we store (see Fig. 2):

- The 3D point $P$ which projects to the center of this pixel

- The polygon ID of the visible surface containing this point

- The form factor $F_{i}$ to each light source from this point

- The visibility $V_{i}$ to each light source from this point

- The estimated surface reflectance $R$ at this point

Actually we create one such data structure for each radiance image plus an additional one for interactive use which also stores the estimated indirect radiance $\hat{B}$ estimated by the radiosity system for this point. The radiance images additionally store a confidence $K_{i}(<=1)$ at each pixel which indicates how reliable we think its reflectance estimate is.

The polygon ID and 3D point $P$ are obtained by using an item buffer [25] and z-buffer depth values. The form factor $F_{i}$ is computed using a standard point-to-polygon technique [1]. The visibility $V_{i}$ is the fraction of the light source which is visible from point $P$ and is estimated by ray casting from the point to the light source. The number of rays is varied adaptively from 4 to 64, with the higher number being used in regions of penumbra.

Initially, confidence $K_{i}$ is set equal to $V_{i}$, since we have less confidence in regions in shadow.

\subsection{Reflectance Recovery Algorithm}

If we assume that our surfaces are diffuse then there is a simple relation between the radiance $L$ seen by a pixel in the camera, the reflectance $R$ at point $P$, and the incident light on point $P$ given by:

$$
L=R\left(\sum_{i} F_{i} V_{i} E_{i}+\hat{B}\right)
$$

where $E_{i}$ is the emittance of light $i, F_{i} V_{i} E_{i}$ is the direct illumination due to light $i$ and $\hat{B}$ accounts for all indirect light. The emittance value is currently set arbitrarily, and an appropriate scaling factor applied to compensate during display.

If all the quantities in question were available and exact, we could estimate each of these quantities and solve exactly for the reflectance at each pixel using, for a given light $i$ :

$$
R_{i}=\frac{T^{-1}\left(C_{i}\right)}{F_{i} V_{i} E_{i}+\hat{B}}
$$




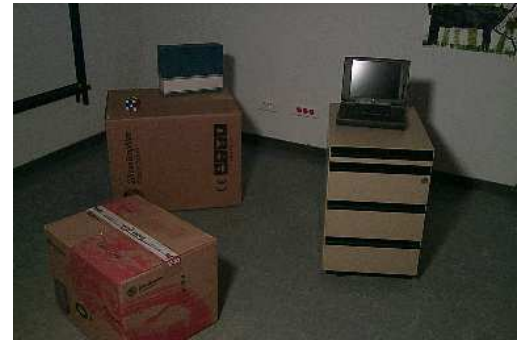

View 1

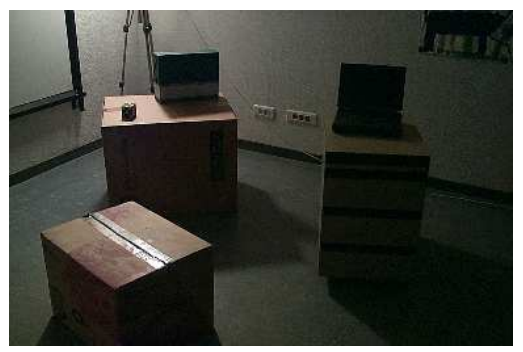

View 2

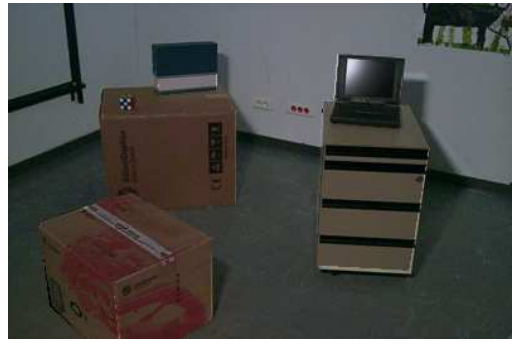

Reflectance 1

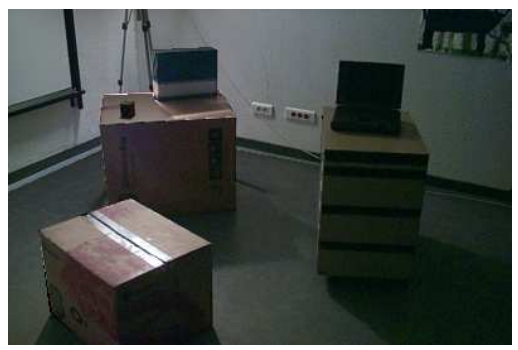

Reflectance 2

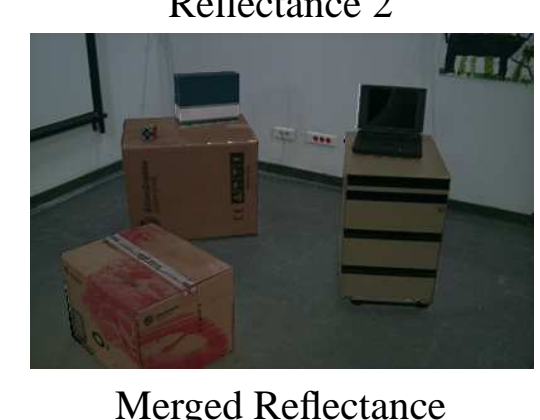

Merged Reflectance

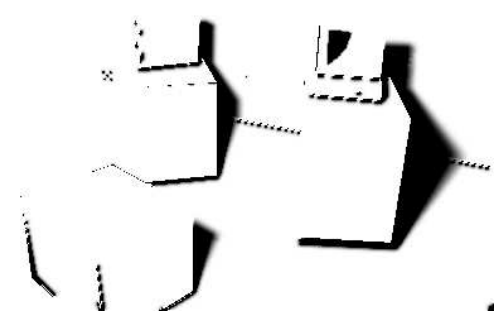

Confidence 1

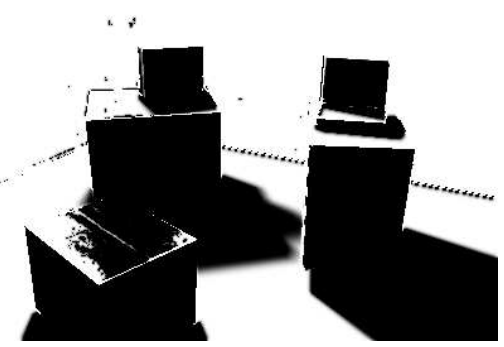

Confidence 2

Figure 3: Two of the seven radiance image views, the confidence (visibility) images, and the resulting reflectance, extracted using Eq.(2). The merged reflectance is shown at the bottom.

where $C_{i}$ is the pixel color recorded by the camera and $T()$ is the response function of the camera. This function was unavailable for our camera ${ }^{1}$ so we have used a simple scaling, though it could be accurately estimated using the method of Debevec and Malik[3].

As a first approximation to indirect lighting $\hat{B}$, we have used an ambient term equal to the average image color times a user specified average reflectance [10]. The resulting reflectance gives satisfactory results for our test cases; nonetheless, it is clear that more involved indirect lighting calculations are necessary in other contexts, where accurate reflectance is paramount. Some experiments were performed with an iterative approach to reflectance estimation using our radiosity solution, without much improvement in the reflectance estimate. Nonetheless, this is clearly a topic of future work.

Because of the many approximations in our system including the geometry, indirect light, and diffuse assumption, we know that our reflectance estimates will sometimes be quite inaccurate (e.g., in shadow regions where the indirect term dominates). We compensate for this by combining the reflectance estimates from multiple radiance images to form a much more robust reflectance estimator.

For each radiance image $i$, we also estimate our confidence $K_{i}$ for each pixel reflectance estimate. The merged pixel reflectance is can be formed by a weighted average of individual reflectance

\footnotetext{
${ }^{1}$ A Kodak DC260 digital camera.
} 


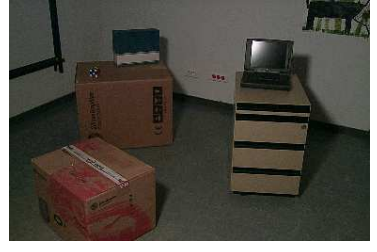

(a)

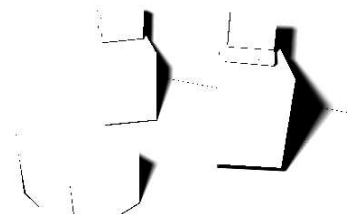

(b)

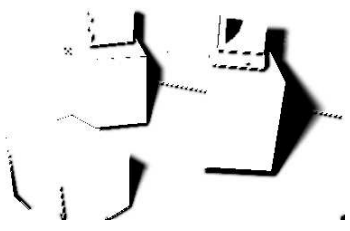

(c)

Figure 4: The filtering process for the confidence images. (a) The original radiance image for given scene and lighting conditions. (b) The original confidence image (= visibility) (c) The final filtered confidence image.

estimates:

$$
R=\frac{\sum_{i=0 . . n} K_{i} \times R_{i}}{\sum K_{i}}
$$

Due to inaccuracies of both the geometric reconstruction and the lighting parameter estimation, this reflectance estimate is not as accurate as required for our purposes. Since we have used $K_{i}=V_{i}$ (visibility) initially, we filter the confidence values $K_{i}$ to compensate for these inaccuracies (see next section). Eq. (3) will then be re-applied with the new confidence values to obtain an improved merged reflectance.

\subsection{Filtering Confidence Values}

When combining the reflectances, we take into account the confidence we have in each pixel reflectance estimate $R$ of Eq. (3). As we saw, this can be achieved by using the visibility computed as an initial confidence value estimate. Near shadow boundaries however, the visibility $V$ depends heavily on the exact geometry configuration and thus may be unreliable due to inaccuracies in our reconstructed model. Reflectance estimates in regions of partial or total shadow are also much less reliable. In addition, the original light source images might contain the image of the (moving) light source fixture. We want to remove this geometry from the images.

The problem of shadow boundary accuracy and the light source fixture geometry is addressed by applying successive filter operations on the confidence images (i.e., an "image" of the $K_{i}(x, y)$ ). The choice of filters and their domain sizes was based on the requirements of each process; we use standard filters such as min, smoothing and outlier.

The first two filters aim at extending shadow boundaries, so that the regions of low confidence become larger. To do this a 5x5 min filter is applied: at each pixel $(x, y)$, a 5x5 window is examined, and the value of $K(x, y)$ is replaced with the minimum. We then apply a $5 \times 5$ smoothing filter, to ensure smooth transitions in the shadow regions. An average $\kappa$ of the $5 \times 5$ window of confidence values is computed. If $\kappa$ is lower than the current confidence, $K(x, y)$ is replaced with $\kappa$. This results in smoothing the transitions between the images used in the merge, since the weighted average is performed with the (smoothed) $K$ values.

To remove the lighting fixtures we apply an outlier filter on the radiance images for each light source position. For each pixel, a table is constructed with the values of reflectance for each image which have been computed using Eq. (2) (we only consider values which have a $K_{i}>\varepsilon$, where $\varepsilon$ is typically around .75). The median $\mu$ of this table is computed, and if $\left|R_{i}-\mu\right|>\varepsilon_{2}$, (where $\varepsilon_{2}$ is another user defined threshold, typically around 0.3 ), then $K_{i}(x, y)$ is set to 0 . A $3 \times 3$ smoothing filter is then applied, again so that transitions between each image during the merge will occur smoothly. 
An example of this process is shown in Figure 4. In Figure 4(b), the original confidence image (initialised to $V_{i}$ ) is shown for the scene with lighting conditions shown in 4(a). In Figure 4(c), the min, smoothing, outlier and second smoothing filters have been applied. Notice that the viewdependent highlight on the laptop screen has been given very low confidence.

Once the filters have been applied, we perform a new merging operation, again using Eq (3). Since we have smoothed and expanded shadow borders, the transitions are less pronounced, and the reflectance computed has fewer artifacts. An example of the merged reflectance is shown in Figure 3 (bottom image).

\subsection{Texture Filling for Real Object Removal}

In order to be able to remove a real object from the scene, we integrated El-Maraghi's [7] implementation of Heeger and Bergen's [13] texture synthesis into our system. By using a technique similar to Igehy and Pereira [14], we are able to fill the gaps left in the image when an object from the reconstructed scene is chosen for removal.

To synthesize the textures needed, we extract a texture sample from the reflectance image from every polygon that now covers the region to fill. The extraction of the sample is currently done manually, but we are experimenting with automatic extraction procedures. This sample is fed to the synthesis algorithm which generates a synthesized texture of the same size as the region to fill. The generated texture is correctly applied to the reflectance using a masking process, described in Section 5.3. The generated textures are stored for objects marked as "removable" accelerating the interactive remodeling operations.

It should be noted that texture generation is performed on the reflectance image and is thus not hindered by shadows or lighting variations during the object removal. The reprojection of the shadows with the new scene will generate a correct image of the scene without the real object.

\subsection{Initializing the Hierarchical Radiosity System}

To bootstrap the hierarchical radiosity system, the reflectance values recovered by Eq. (3) are reprojected onto the corresponding polygons, initialising the reflectance values. For the polygons not visible in the image used for the interactive session, we take a sample of the texture during the the photomodeling session and use the average value using Eq. (2). This also true for the hidden parts of polygons which are actually visible.

With this approximation, a first radiosity solution is computed by our system, using an implementation of hierarchical radiosity with clustering [23]. The subdivision is set to a relatively coarse level since such a level is sufficient for indirect light only, which varies slowly. An example mesh is shown in Figure 5(b).

Recall that direct effects, including direct shadows, are treated separately for display. Direct light is however computed by the radiosity system, but simply ignored for display. The subdivision is fixed at the beginning of the process to a minimum area threshold. Nonetheless, we maintain the hierarchical nature of the radiosity algorithm, since links are established at different levels of the hierarchy, using a "standard" BF refiner [12]. Thus we will only need to update links and the radiosity values when performing interactive modifications. 


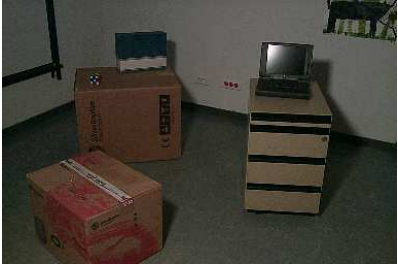

(a)

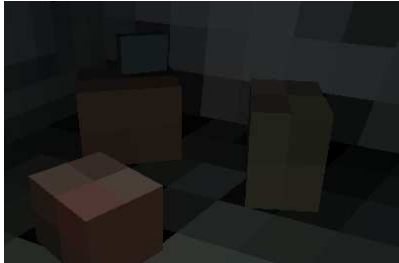

(b)

Figure 5: (a) The original view of the scene and (b) the corresponding radiosity mesh used to simulate indirect light and dynamic updates; note the coarse subdivision.

\section{Interactive Modification of Scene Properties}

Once the reflectance has been computed for each pixel and the radiosity system set up, we can perform interactive modification of scene properties. The modifications which our system permits are related to lighting and geometry. The former includes changing a real light or adding virtual lights; the latter includes adding and moving virtual objects and removing real objects.

All the results described below are best appreciated by watching the accompanying videotape of an interactive virtual relighting/remodeling session. All timing results reported below have been taken on a SGI R10000 195Mhz processor. The web page ${ }^{2}$ contains better images and additional companion information.

\subsection{Modifying Illumination}

When we modify a light source emittance, two operations need to be performed:

- For indirect illumination, we need to compute a new radiosity solution. Given that the subdivision and the link structure is fixed after the initial solution, updating indirect illumination simply requires a few successive sweeps of the hierarchy to "gather" and "push-pull" [12] radiosity and is very fast (less than .05 seconds in our test scenes).

- For display, the direct lighting component is recomputed at each pixel. Indirect illumination is displayed using hardware smooth-shading of the elements of the hierarchical radiosity subdivision, which are then blended into the final image. This results in the addition of $\hat{B}$ at each pixel.

In the pixel structure, we have stored the visibility and form factor with respect to each light source. Thus the computation of the direct component is very rapid.

When displaying an image, we compute the following color at each pixel:

$$
C=R\left(\sum_{s=0 . . n_{s}} F_{s} V_{s} E_{s}+\hat{B}\right)
$$

for the $n_{s}$ (real or virtual) light sources in the scene. Thus shadows are reprojected due to the visibility term $V_{s}$, since they have been removed from the reflectance.

An example is shown in Figure 6. The original photo is shown in (a), reprojected initial lighting conditions in (b), and we show the addition of a virtual light source in (c). The entire update for adding

\footnotetext{
${ }^{2}$ http://www-imagis.imag.fr/Membres/Celine.Loscos/relight.html
} 


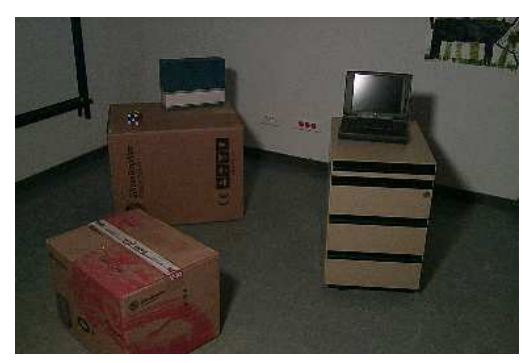

(a)

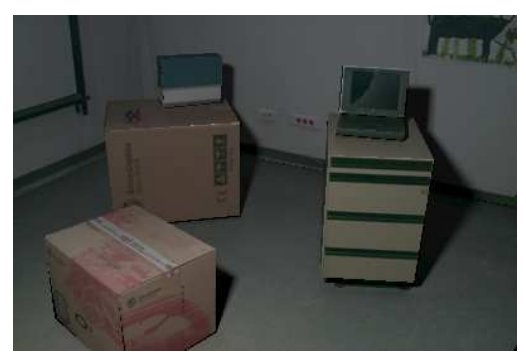

(b)

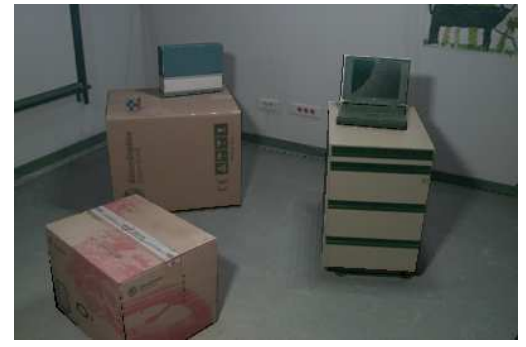

(c)

Figure 6: (a) The original radiance image (photo) (b) original reprojected lighting conditions, displayed using the recomputed direct and indirect components, (c) a virtual light has been inserted into the scene adding the light took 3.1 seconds (for 400x300 resolution).

the virtual light takes 3.1 seconds broken down as follows: visibility $2.5 \mathrm{~s}$, shaft/radiosity operations $0.4 \mathrm{~s}$., indirect light blending and other $0.2 \mathrm{~s}$. Recall that in the case of the light source insertion, we are required to update all the pixels of the image. During dynamic updates, we cast a small number of rays to the light sources, resulting in aliased shadows. An additional "shadow clean-up" could be performed when the user stops modifying the scene, with a higher shadow sampling rate.

\subsection{Modifying Scene Geometry}

To allow interactivity when adding, removing or moving objects and lights, we maintain a shaft data structure [11], inspired from the work of Drettakis and Sillion [6]. Updating the entire table requires in the order of a few minutes for visibilities, especially when using many rays per light source; using the method described below reduces this time to fractions of a second.

A hierarchical shaft [11] data structure is constructed from the first radiosity solution, corresponding to each link. When we add an object it is first attached to the root cluster of the scene; links are established to the light sources as appropriate, based on the refinement criterion, and visibility information is computed.

The hierarchy of shafts is used for two purposes: (a) to identify the pixels for which direct illumination has changed (i.e., the shadow regions of the new object); and (b) to identify the links for which the form-factor needs to be modified (i.e., all links whose shaft is cut by the new object), for both direct and indirect light transfers.

To achieve the above, we descend the hierarchy of shafts, finding those intersected by the new object. The hierarchical elements attached to the end of a shaft originating at a light source are marked as "changed". While descending, the form-factors of the modified links are updated (i.e., a visibility calculation is performed). With all necessary links updated, we recompute a radiosity solution with only gather and push-pull steps.

The pixel data structure is then updated and displayed. The bounding box of the initial and final position of the moving object are first projected onto the image-plane, limiting the region of the screen directly affected by the motion. For this region an new item buffer is performed, and the pixels under the previous object position are found as well as those under the new position, since the polygon IDs will have changed. For these pixels, a reflectance is updated to the original value for the "uncovered" pixels and to that of the virtual object for the newly covered pixels. New form-factors and visibility values are then computed for all the pixels changed in the modified region.

For the pixels associated with patches tagged as "changed", visibility with respect to the source is recomputed. These are not as localized as the directly affected pixels, but their number is often small.

RT n ${ }^{\circ} 0230$ 


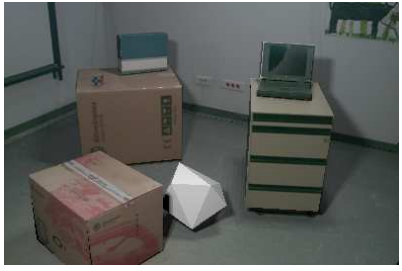

(a)

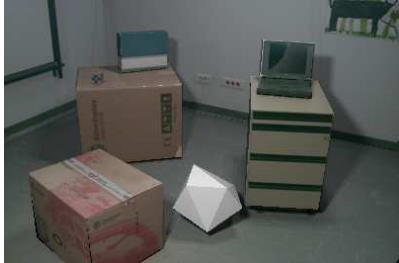

(b)

Figure 7: (a) A virtual object has been inserted into the scene with both lights on; adding the object required $1 \mathrm{sec}$. (b) moving the virtual object requires $1.1 \mathrm{sec}$.

The entire pixel table is then traversed to update the indirect illumination value at each pixel, based on the new global illumination calculation; again, this is performed with hardware rendering of the hierarchical radiosity patches.

When inserting a new light source, the form-factor and visibility with respect to the source need to be computed for every pixel.

When removing an object, we perform a similar process. We first remove every link of the removed object, then delete all the corresponding shaft structures.

When moving an object, the process is equivalent, but we do not have to delete the links. We just have to update the information (form factors and visibilities). Shafts due to the moving object are deleted and reconstructed with its new position.

In Figure 7(a) we show the insertion of a virtual object, which requires $1 \mathrm{sec}$, of which visibility accounts for $.5 \mathrm{sec}$, shafts $.1 \mathrm{sec}$ and the rest $.4 \mathrm{sec}$. When moving the virtual object, we achieve update rates of about $1 \mathrm{sec}$ per frame, with a similar breakdown to that of the object insertion (Figure $7(b))$.

\subsection{Removing Real Objects}

When the user chooses to remove an object, she indicates the object to the system. Similarly to virtual objects, since the correspondences between polygons and pixels are known through the polygon IDs stored in the pixel data structures, we know exactly which region of screen will have to be filled. We automatically create two masks corresponding to this region: a weight mask and a texture mask [14]. At first, each contains " 1 " over the region to fill and " 0 " elsewhere. We extend the weight mask a few pixels to compensate for inaccuracies in the removed object geometry (to avoid leaving any color from the removed object in the image).

The object is then removed from the scene and a new item buffer is performed to update the polygon IDs. The polygon IDs present in the region to be filled indicate from which polygons we have to extract textures. The texture mask is filled with these new IDs and the weight mask is blurred around its " $0 / 1$ " borders. This allows the composition of the synthesized texture with the texture from the image: when the mask is 0 , the color of the pixel will be the color in the reflectance image, when the mask is 1 the color will be taken from the synthesized texture and a fractional weight will allow a smooth transition from the synthesized texture to the original image (e.g. the original colors present in the image).

The reflectance is then updated for the pixels affected, as well as the visibility and form-factors, as in the case of virtual object motion/removal. Results of object removal are shown in Figure 8.

A second example of real object removal is shown in Figure 9. In the context of an interior redesign, we may want to remove doors for example, which is hard to do in the real world. This is 


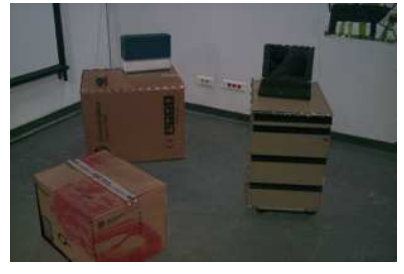

(a)

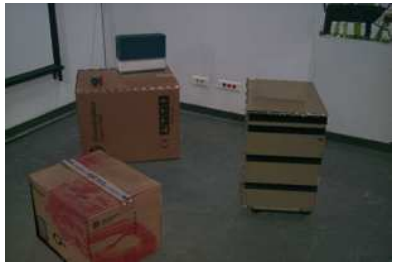

(b)

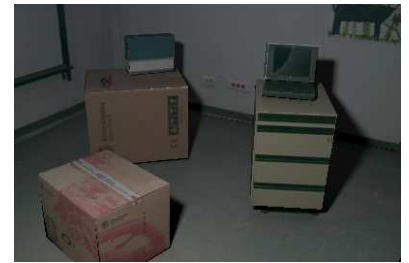

(c)

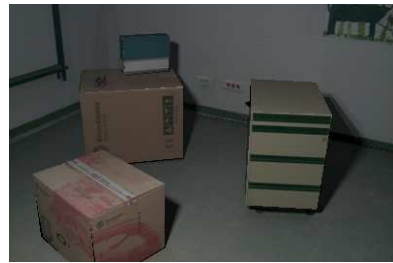

(d)

Figure 8: Texture filling examples for real object removal. (a) Initial reflectance image (b) The laptop is removed. The laptop was removed entirely synthetically since no additional image was captured. (c) The original relit image, (d) the relit image after removal. Removal of the laptop took $0.7 \mathrm{~s}$, since generated textures are pre-computed for "removable" objects.

shown Figure 9(b). Note that due to approximate reflectance estimation, the texture generation results in slightly visible discontinuities. A virtual object has been added in (c) and a different lighting configuration created in (d).

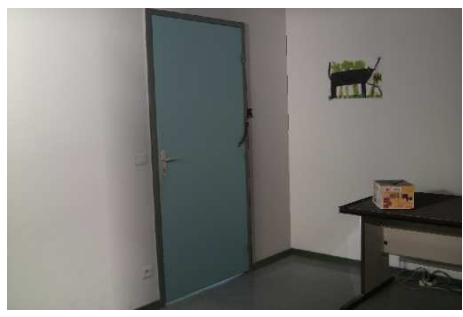

(a)

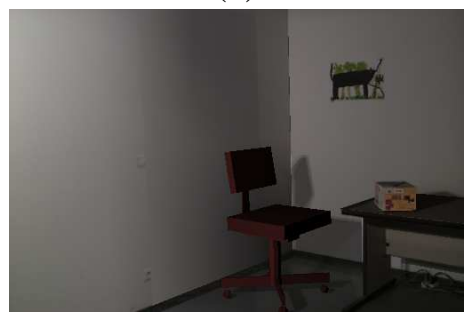

(c)

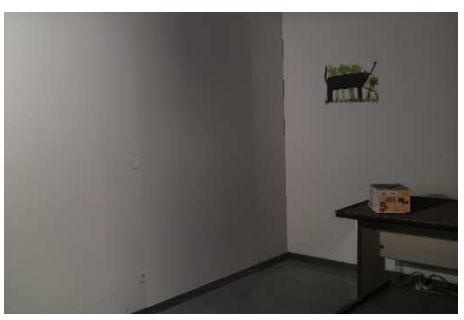

(b)

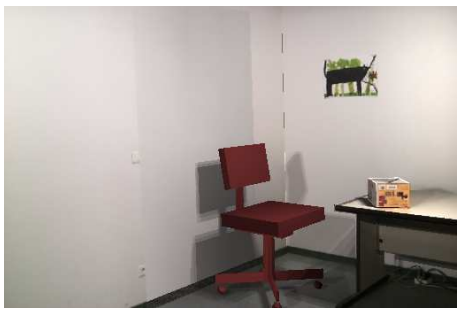

(d)

Figure 9: A second real object removal example. (a) The original relit image, (b) the relit image after removal of the door. The removal took $2.9 \mathrm{~s}$, for a resolution of 512x341. (c) A virtual chair has been added to the scene, requiring $3.4 \mathrm{sec}$, and (d) a virtual light added (needing $6.6 \mathrm{~s}$. ).

\section{Conclusion}

We have presented a new approach to synthetic relighting and remodeling of real environments. Our approach is based on a preprocessing step to recover approximate reflectance properties from a sequence of radiance images. Radiance images are taken from a fixed viewpoint with varying illumination (i.e., different positions of the same light source), using a simplified reconstructed model of the scene. Using the information in the images and the 3D reconstructed model, we create reflectance images for each light position by estimating direct illumination and light source visibility as well as indirect light. The reflectance images are merged by a weighted average based on the confidence level we have in the reflectance at each pixel in each radiance image. In our case, this is based on 
visibility (points in shadow have low confidence); a filtering step is applied to compensate for errors in geometric reconstruction and illumination computation.

After the reconstruction has been performed we can interactively modify scene properties. This is achieved by efficiently identifying regions of the screen which need updating, and performing a pixelby-pixel update for direct light. Indirect lighting is treated separately with an efficient hierarchical radiosity structure, optimized for dynamic updates.

In our implementation we can virtually modify real light intensity, insert and move virtual objects, and even remove real objects interactively. Despite inevitable artifacts, the quality of the images is sufficient for the purposes of interactive lighting design and limited remodeling.

Independently to our work, Yu, Debevec and Malik [26], have developed more robust techniques for reflectance estimation, including specular effects in particular. These are based on capturing images of the entire scene, and using radiosity to estimate the reflectance using clever iterative methods and high-dynamic range images. We believe that our approach can benefit from such improved reflectance estimation (for example to remove the artifacts in texture generation in Figure 9) as well as for the reflectance of objects which are not visible in the radiance image. On the other hand, we believe that both our interactive approach, especially for global illumination, as well as our confidence maps could be useful for such approaches.

In future work, using the high dynamic range radiance images of Debevec and Malik [3] will allow us to achieve more accurate reflectance extraction. Once we have more confidence in the original radiance most of the error in the reflectance estimation will be due to indirect light. The hierarchical radiosity framework has the added advantage that it can be used to bound indirect illumination errors and thus should allow us to achieve better results.

We also need to investigate ways to allow motion of the viewpoint, which is currently an important limitation of our approach. Also, the texture generation approaches we have used are limited to stochastic textures. With some user intervention, it may be possible to achieve satisfactory results with deterministic textures also.

From a more practical point of view, we can add the synthetic motion of real objects simply into our system. A view-independent texture of the real object is required, which can be provided by our photomodeling system, as well as a modified rendering routine. As was discussed in the results, the largest expense in the updates is the calculation of visibility for direct lighting. These calculations can be easily parallelized, and we hope to achieve good speedups in a parallel version, enhancing interactivity.

\section{References}

[1] D. R. Baum, H. E. Rushmeier, and J. M. Winget. Improving radiosity solutions through the use of analytically determined form-factors. In Jeffrey Lane, editor, Computer Graphics (SIGGRAPH '89 Proceedings), volume 23, pages 325-334, July 1989.

[2] P.E. Debevec. Rendering synthetic objects into real scenes: Bridging traditional and imagebased graphics with global illumination and high dynamic range photography. In SIGGRAPH 98 Conf. Proceedings, Annual Conf. Series, pages 189-198, July 1998.

[3] P.E. Debevec and J. Malik. Recovering high dynamic range radiance maps from photographs. In SIGGRAPH 97 Conf. Proceedings, Annual Conf. Series, pages 369-378, August 1997. 
[4] P.E. Debevec, C.J. Taylor, and J. Malik. Modeling and rendering architecture from photographs: A hybrid geometry- and image-based approach. In SIGGRAPH 96 Conf. Proceedings, Annual Conf. Series, pages 11-20, 1996.

[5] G. Drettakis, L. Robert, and S. Bougnoux. Interactive common illumination for computer augmented reality. In Eurographics Workshop on Rendering 1997, pages 45-56, June 1997.

[6] G. Drettakis and F. Sillion. Interactive update of global illumination using A line-space hierarchy. In SIGGRAPH 97 Conf. Proceedings, Annual Conf. Series, pages 57-64, August 1997.

[7] T. El-Maraghi. "an implementation of heeger and bergen's texture analysis/synthesis algorithm" with source code. http://www.cs.toronto.edu/tem/2522/texture.html.

[8] O. Faugeras. Three-Dimensional Computer Vision - A Geometric Viewpoint. MIT Press, 1993.

[9] O. Faugeras, S. Laveau, L. Robert, G. Csurka, and C. Zeller. 3D reconstruction of urban scenes from sequences of images. Technical report 2572, INRIA Sophia-Antipolis, May 1995.

[10] A. Fournier, A.S. Gunawan, and C. Romanzin. Common illumination between real and computer generated scenes. In Proc. of Graph. Interface'93, pages 254-262, May 1993.

[11] E. A. Haines and J. R. Wallace. Shaft Culling for Efficient Ray-Traced Radiosity. In P. Brunet and F. W. Jansen, editors, Photorealistic Rendering in Computer Graphics (Proceedings of the Second Eurographics Workshop on Rendering), New York, NY, 1994. Springer-Verlag.

[12] P. Hanrahan, D. Salzman, and L. Aupperle. A rapid hierarchical radiosity algorithm. In Thomas W. Sederberg, editor, Computer Graphics (SIGGRAPH '91 Proceedings), volume 25, pages 197-206, July 1991.

[13] D.J. Heeger and J.R. Bergen. Pyramid-Based texture analysis/synthesis. In SIGGRAPH 95 Conf. Proceedings, Annual Conf. Series, pages 229-238, August 1995.

[14] H. Igehy and L. Pereira. Image replacement through texture synthesis. In Proceedings of the 1997 IEEE International Conf. on Image Processing, 1997.

[15] F. Leymarie et al. Realise : reconstruction of reality from image sequences. In International Conf. on Image Processing, pages 651-654, Lausanne (Switzerland), sep 1996. IEEE Signal Processing Society.

[16] C. Loscos and G. Drettakis. Interactive relighting of real scenes. Technical report 0225, INRIA Rhone-Alpes, November 1998.

[17] C. Loscos, G. Drettakis, and L. Robert. Interactive modification of real and virtual lights for augmented reality. In SIGGRAPH 98 Technical Sketch (Visual Proceedings), July 1998.

[18] E. Nakamae, K. Harada, T. Ishizaki, and T. Nishita. A montage method: The overlaying of the computer generated images onto a background photograph. In David C. Evans and Russell J. Athay, editors, Computer Graphics (SIGGRAPH '86 Proceedings), volume 20, pages 207-214, August 1986.

[19] Photomodeler. http://www.photomodeler.com.

$\mathrm{RT} \mathrm{n}^{\circ} 0230$ 
[20] P. Poulin, M. Ouimet, and M.-C. Frasson. Interactively modeling with photogrammetry. In Eurographics Workshop on Rendering, pages 93-104, June 1998.

[21] Y. Sato, M.D. Wheeler, and K. Ikeuchi. Object shape and reflectance modeling from observation. In SIGGRAPH 97 Conf. Proceedings, Annual Conf. Series, pages 379-387, August 1997.

[22] E. Shaw. Hierarchical radiosity for dynamic environments. Computer Graphics Forum, 16(2):107-118, 1997.

[23] F. X. Sillion. A unified hierarchical algorithm for global illumination with scattering volumes and object clusters. IEEE Transactions on Visualization and Computer Graphics, 1(3):240-254, September 1995. ISSN 1077-2626.

[24] G.J. Ward. The RADIANCE lighting simulation and rendering system. In Proceedings of SIGGRAPH ’94, Annual Conf. Series, pages 459-472, July 1994.

[25] H. Weghorst, G. Hooper, and D. P. Greenberg. Improved computational methods for ray tracing. ACM Transactions on Graphics, 3(1):52-69, January 1984.

[26] Y. Yu, P.E. Debevec, J. Malik, and T. Hawkins. Inverse global illumination: Recovering reflectance models of real scenes from photographs. In to appear at SIGGRAPH '99, August 1999.

[27] Y. Yu and J. Malik. Recovering photometric properties of architectural scenes from photographs. In Computer Graphics (ACM SIGGRAPH'98 Proceedings), 1998. 
Unit'e de recherche INRIA Lorraine, Technop^ole de Nancy-Brabois, Campus scientifi que, 615 rue du Jardin Botanique, BP 101, 54600 VILLERS LÈS NANCY

Unit'e de recherche INRIA Rennes, Irisa, Campus universitaire de Beaulieu, 35042 RENNES Cedex

Unit'e de recherche INRIA Rh^one-Alpes, 655, avenue de l'Europe, 38330 MONTBONNOT ST MARTIN

Unit'e de recherche INRIA Rocquencourt, Domaine de Voluceau, Rocquencourt, BP 105, 78153 LE CHESNAY Cedex

Unit ${ }^{\prime}$ de recherche INRIA Sophia-Antipolis, 2004 route des Lucioles, BP 93, 06902 SOPHIA-ANTIPOLIS Cedex

Éditeur

INRIA, Domaine de Voluceau, Rocquencourt, BP 105, 78153 LE CHESNAY Cedex (France)

http://www.inria.fr

ISSN 0249-6399 